International Journal of Engineering \& Technology, 7 (4.6) (2018) 185-192
International Journal of Engineering \& Technology
SPC
Website: www.sciencepubco.com/index.php/IJET
Research paper

\title{
A Analysis of Machine Learning in Wireless Sensor Network
}

\author{
Bhanu chander $^{1 *}$, Prem Kumar.B ${ }^{2}$, Kumaravelan ${ }^{3}$ \\ ${ }^{1}$ Rearch Scholar, Dept of Computer Science, Pondicherry University, India \\ ${ }^{2}$ Rearch Scholar, Dept of Computer Science, Pondicherry University, India \\ ${ }^{3}$ Assistant Professor, Dept of Computer Science, Pondicherry University, India \\ *Corresponding author E-mail:gujurothubhanu@gmal.com
}

\begin{abstract}
Advances in hardware as well as wireless network tools have positioned us at the doorstep of a new-fangled era where undersized wireless devices will endow with access to information every time, everyplace plus enthusiastically contribute in constructing smart atmosphere. The sensors in WSN's assemble information regarding the substances they are exploited to sense. Nevertheless these sensors are restricted in their performance by restrictions of power plus bandwidth. Machine Learning methods can facilitate them in overcoming such restrictions. During the past decade, WSNs have seen progressively more rigorous implementation of highly developed machine learning algorithms for information handing out and improving network performance. Machine learning enthuse countless realistic clarifications that make best use of resource exploitation along with make longer the existence of the network. In particular, WSN designers have effectively agree to machine learning paradigms to deal with widespread purposeful problems associated to localization, data aggregation, fault detection, Security, node clustering, prediction models and energy aware routing, etc.
\end{abstract}

Keywords: Wireless sensor network, Machine learning, Energy efficient, Security, Data aggregation

\section{Wireless sensor networks}

Wireless sensor network has improved significantly from the last decade with the huge change in electronics and communication and become the solitary of the popular exploration domain. Wireless sensor network is collection of small, tiny sensor nodes collects sensed information and transmitted to end users or decision makers. Sensor nodes continued sensing, event detection, location detection and some characteristics like fault tolerance, rapid deployment, self organization makes them to use many critical scenarios like Military surveillance, Home automation, Target tracking, Health monitoring, Sales tracking, Fire detection, Internet of Things, Sea water levels, Volcano observations. These applications required perfect and absolute data for taking decisions. Thousands or countless sensors are deployed in a violent environment to supervise, collect sensed information to sink node. Sensor nodes densely deployed, individual sensors collaborate interact with other sensor nodes over wireless transmission medium frequently radio links. Sensor nodes have limited computational power, energy resources with their small size and another huge problem is environment which they deployed changes rapidly over time. Analysis of data collected from sensors at timely manner is highly important. Untreated data collected from sensor node is in-accurate and in-completeness [1.2].

With outspread range of applications from Military surveillances to smart building technologies are improved by deploying the WSN's expertise. Low cost, low energy, easy deployment, high rage sensing and self organization are some of the advantages provided by WSN's. However the advantage provided by WSN's comes with great range of challenges. Most of the challenges are connected with characteristics of a wireless sensor network fol- lows as development of large scale network which stand to Scalability, ability to handle with node collapses, Node communication failures, Mobility status of sensor node, Energy devour restrictions to node using batteries or energy consequence and competence to stand disciplinary natural circumstances [3.4].

Wireless senor networks mainly suffered from power resources, to reduce power consumption there are many routing algorithms are invented. In WSN transmit data from source-sink node or sinksensor node is described as routing. Rather than routing, to design a wireless sensor network designer should take care about challenges like localization, deployment, scheduling, security, data aggregation, Quality of services. Every senor node introduce large amount of data for transmitted, processed and received to WSN. Processing and making decisions is impossible due to the sensor limited energy and bandwidth constraints. So there is necessitate of pertain Machine Learning [5.6].

\section{Machine learning}

Artificial intelligence $(\mathrm{AI})$ has been a captivating perception of science fiction for decades; Scientists have made breakthroughs in "machine learning" using neural networks which impersonate the processes of genuine neurons. Artificial intelligence can predominantly define the machine has ability to solve a problem without human involvement.AI illuminating that data generate a solution for itself. Machine learning capture the approach to sophisticated level by providing data vital for a machine to train and modify suitable when exposed to new data and produce more effective results. Machine learning is a paradigm that refers to learning from ancient times experience to enhance future performance. The solitary focal point of this field is automatic learning [7.8.9]. 
Machine learning expertises are used immeasurable for an ample scale of exercises incorporates classification, regression furthermore density estimation in heterogeneity of utilization territories such as bioinformatics, speech detection, spam recognition, computer perspective or visualization and fraud detection along with broadcasting networks. The algorithms as well as competence take advantage of emerge from numerous miscellaneous fields together with mathematics, computer science, statistics and neuroscience. Machine learning is employ to instruct machines how to knob the data more proficiently. After collecting the data we can't realize the accurate information. In these types of cases we use machine learning. Many industries like medicine, military etc apply machine learning to unsheathe functional information. The main benefit of using machine learning is once an algorithm grasp what to accomplish with data; it can accomplish that work mechanically. Emergent handling of machine learning technologies in computerization of WSN operations makes them skilful and professional [9.10.11.12].

\section{Machine learning: Definition}

Machine learning enlarge a computer model for learning process and provides the finest solution for the problem by using possession information and enlarge progress of systems.

"The advocacy methods improving the machine performance of detecting and describing unpredictability prototype in data".

Machine learning is important in WSN for the following reasons [13.14.15.16]:Wireless sensor networks deployed in rough environment where traditional networks are failed to deploy, Number of assignments in WSN arranged by some mathematical replica like routing, data aggregation etc. under related situations machine learning valuable to provides low complexity, computational cost for the system(1). Wireless sensor nodes due to enormous amount of region sensors collect huge amount of data and send it to base station in addition to extracting important information from huge data is complicatedness at base station as well as at sensor node. An often WSN application comes with some degree of data coverage which reduce the power handling with the help of machine learning models to maximum data coverage with superior sensor deployment(2). Sensor networks usually installed in rough environments which modifies regarding time for example we installed sensor nodes for real time application when sensor nodes changes or moves from one place to another that will affect the result. So it's pleasing to develop sensor networks which can be control powerfully in those environments (3).

From the past decade machine learning algorithms are broadly apply in wireless sensor networks for getting better results in processing. Machine learning has a massive number of algorithms and premises applying these algorithms premises to WSN applications to provide remarkable assistance [10.14].

There are many existing algorithms in Machine learning algorithms all are come under the models of supervised learning, unsupervised learning and Reinforcement learning. Here supervised learning first training data set is prepared and evaluate with the testing data. Here an algorithm learns some techniques from training data set and apply on testing data set for classification or taxonomy. Unsupervised learning algorithms there is no training data, algorithms categorize the example data set into different classes based on their related features. Reinforcement learning algorithms an agent learns from its environment similar to which actions to take such that conclusion is more optimistic [11.12.15].

\section{Supervised Machine Learning}

In Supervised Machine Learning, an established advance inputs and output values are used to build the system model. For instance input variable (a) output variable (b) and design a learning algorithm to learn mapping function from input to the output $\mathrm{Y}=\mathrm{f}(\mathrm{a})$. The intention toward guesstimate mapping function hence once we occupy most recent participation data (a) be able to expect the output variable (b) for that data. When designing supervised machine learning we have to remind some steps (1) abuser should know what type of data is used as training set. (2) The training data represents the real world function. (3) Learning algorithm should represent one desired structure. (4) Determine the key aspects from training data set. (5) Finally once selection of parameters and structure determine the accuracy of learning algorithm whether the algorithm mapping the output variable as we estimate this could be done by measuring with the test set that is separate from training data set [9.10.11.12].

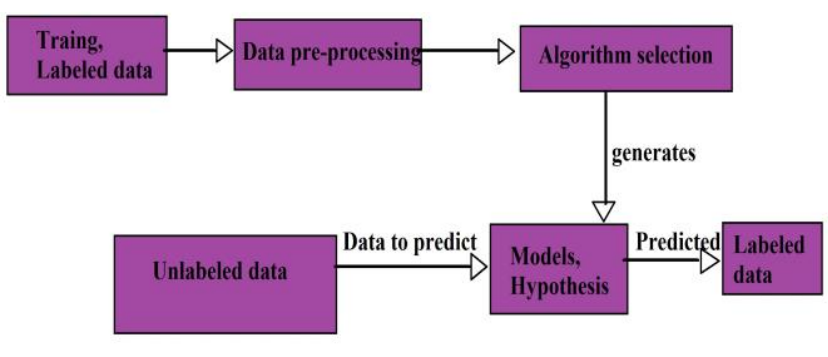

Fig. 1: Supervised learning process

Supervised ML algorithms widely used in WSNs Approaches to solve many challenges such as objects targeting, localization query processing, event detection, quality of services, intrusion detection, security. Most famous supervised learning algorithms are listed below:

\subsection{Decision tress}

Decision trees are one of the predictive models. It systematizes the data by taxonomy pedestal on their principles. Each decision tree contains nodes and branches, where branch is referred to the value that can be take and nodes referred to attributes in a group that can be classified. In this procedure the principles are compared relative to the decision condition that reaches the particular class. Decision trees are mainly used for classification process. Decision trees express as arrangement of mathematical and computational procedure to help explanation, classification of given data set. $(\mathrm{n}, \mathrm{M})=(\mathrm{n} 1, \mathrm{n} 2 \ldots \mathrm{nk} . \mathrm{M})$ Here $\mathrm{M}$ is target variable that we want to describe and $n 1, n 2 \ldots n k$ are the principles that we are taken for task. Decision tress algorithms resolve many unsolved problems in wireless sensor networks design like link loss rate, reliability, restore, corruption rate, mean time to failure [11.12.17.18.19.20].

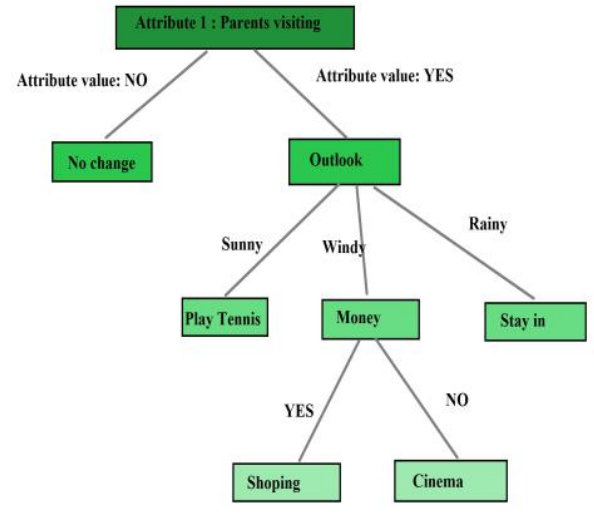

Fig. 2: Decision trees

Wireless sensor network characteristics made them to apply any kind of application. One of the applications is environmental monitoring. A. Mainwaring and D.Culler et al (17) in "Wireless sensor networks for habitat monitoring" addressed overview of sensors used in environmental monitoring. E.Nadimi, T. Bak et al (18) in "Zigbee based wireless sensor networks for classifying the behav- 
ior of herd animals using classification trees" proposed a method to perfectly categorize the exact activities of heard creatures using Decision trees taking consideration of its neck angle, movement of velocity. In animal behavioral, wirelesses sensor networks are widely used because they consider minimum parameter which makes simple implementation and low complexity. Z.Meri, M.Bayoumi et al (19) in "A lightweight fault tolerant target location system for wireless sensor networks" described a method for exact location of target by using time difference of arrival. E.Cayiric, Y.Dogan et al (20) in "wireless sensor networks for underwater surveillance" employed a method for submarine uncovering underwater. Each node predetermined with a cable to surface guide, apiece node come across its setting in 3D space stand on the guiding sensor coordination. Collected data from guided nodes transmitted to the central unit and apply decision tree classification applied on received data to find there is any submarine in located places. Decision trees used to elect cluster head based on battery level, degree of mobility as input vectors to build tree.

\subsection{K- Nearest Neighbour}

k-NN machine learning algorithm classifies data based on the values of near data samples. Here $\mathrm{K}$ is a user defined value, scepticism or trial point is catalog through turning over the label which is a good number frequent amid the $\mathrm{k}$ training examples adjoining to the scepticism point. It's mainly exploiting for classification and regression. Training data is introduces to the learner and then we introduce the new data to learner, learner compare both data values. $\mathrm{K}$ most associated data is taken from priming data set along with greater part of $\mathrm{k}$ taken as new class from new data set. $\mathrm{K}$ nearest neighbor functions with nearest points so computation cost is low which afford more functioning for sensor networks. Omitted sense of sensor node can be calculated by using the average distance between nearest neighbor nodes surrounded by a definite limit. In WSN, Query processing subsystem function is prepared through $\mathrm{k}$ nearest neighbor algorithm [12.13.24, 25].
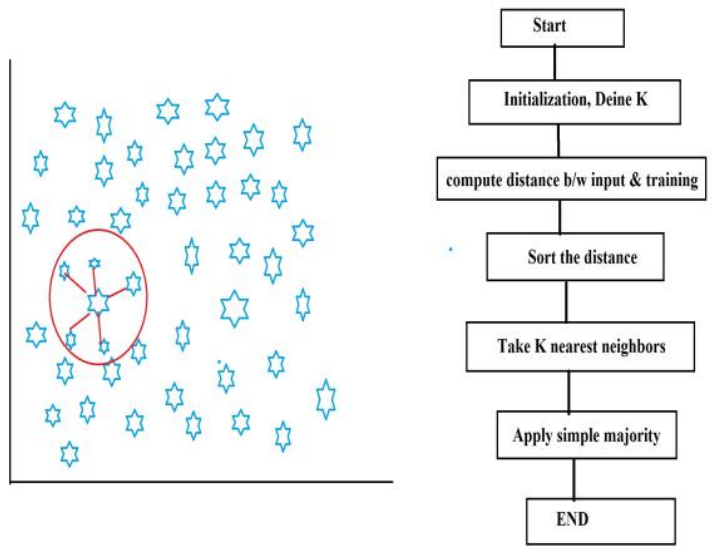

Fig. 3: K- Nearest Neighbor

J.W. Branch, C.G. Gannella et al (21) in "In Network outlier detection in wireless sensor networks" introduced a process via kNN several mislaid networks appraisals calculated by average of its neighbor nodes knowledge. Most highly applicable area of $\mathrm{k}$ nearest neighbor in wireless sensor network is query processing but problem in nearest neighbor algorithm is it's requires to store large amount of data from its environment. J. Winter, W.C Lee et al (22) in "energy efficient processing of k nearest neighbor quires in location aware sensor networks" developed a k nearest neighbor boundary tree algorithm. When node outside boundary location and gets query from application administrator it do regulate its k$\mathrm{NN}$ region. This query processing extended to $3 \mathrm{D}-\mathrm{KNN}$ developed by Jayaraman et al. These processes restrict query processing in 3D space where K-NN nodes deployed. D.Li, K.Wong et al (24) in "Detection, classification and tracking of targets" described single target using $\mathrm{k} N \mathrm{~N}$ concept.

\subsection{Neural networks}

Neural network is derivative from biological concept of neurons. Each neuron connection sent signal from one neuron to another, receiving neuron processed on signal and then signal neurons connected to another or received neuron. Neural networks can be appropriate to seven classes of problems: prototype cataloging, process control, function approximation, clustering, prediction, and optimization furthermore retrieval by content. In WSN using of neural networks there is still no omnipresent owing to soaring computational requirements for learning knowledge with reference to set of connections. Neural networks in WSN mainly used to fine the location of sensor node by Received signal strength, angle of propagation and distance of measurements [13.15.27.28.51].

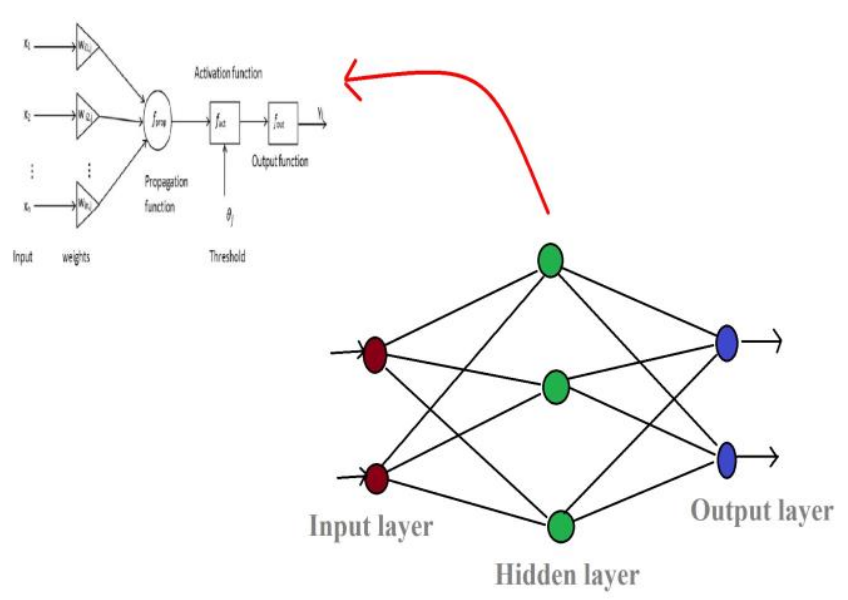

Fig. 4: Artificial Neural networks

WSN vigorously designed for rescue and fire detection. N.Wang, L. Yu et al (25) in "real time forest fire detection using wireless sensor networks" addressed real time forest fire detection make use of neural networks. Nodes sense surrounding environment and collocated information sends to cluster head, cluster head process the data using neural network and only important information is sent to decision makers. O. Postolache, J.Peria, et al (26) in "Smart sensor networks for air quality monitoring application" presented a method for air quality by using neural network approach, at the same time as eliminating temperature, humidity on sensor nodes. Y. Gao, Y.Lin et al (27) in "Wireless sensor network based I-Matrix to achieve lighting control" developed a new approach to light systematize inside elegant construction exploiting neural network algorithm. Neural network uses Illuminated Matrix for appraise the grade of luminance within the lighted quarter. P.Rastogi, A.Snow, et al (28) in "Assessing dependability of wireless sensor networks using neural networks" designed a method to guesstimate sensor node steadfastness metric utilizing neural network. Steadfastness which represents the availability, reach ability, survivability, maintainability of sensor node. Neural networks also used to find the energetic activities as well as their causes on supplementary nodes. DoS attack is kind of attack which targets by flood huge number of fault message traffic, therefore prevents delivery of constructive data. R.Kulakarni, G.Venagayamoorthy et al (29) in "neural network based secure MAC protocol for wireless sensor network" employed a method of neural network prevent the untruthful flooding messages by investigating PRR (packet request rate), APW (average packet waiting time).For reducing transmission collision, processing time. Y.J.Shen, M.Wang et al (30) in "Broadcast scheduling in wireless sensor network using fuzzy Hopfield neural networks" Introduced a time separation multiple access protocols make sporadic instance outline to split the medium access of divergent nodes. Central unit distribute the time slots for each sensor node within the network. 


\subsection{Support vector machine}

SVM procedure associated learning algorithm most extensively used in machine learning for data classification. It divides the data into two separate classes based on the margin calculation. SVM representation is a demonstration the pattern as points in space, plotted so pattern of the unfasten class is estranged with apparent gap that is as spacious as possible. Innovative paradigms are then resembled into that same space plus predicted to be in the right place to a model essentially on which region of the space they sink. In WSN this approach is used to find the malicious behavior nodes by inspect spatial and temporal correlation of data. SVM makes the observations of WSN as points as characteristic space and divides space into segments. These segments estranged as spacious as feasible partition gaps. Innovative analysis will be organized based on which region of the gap they sink. Possible applications of SVM in wireless sensor networks are localization, security [13.15.32.33.34].

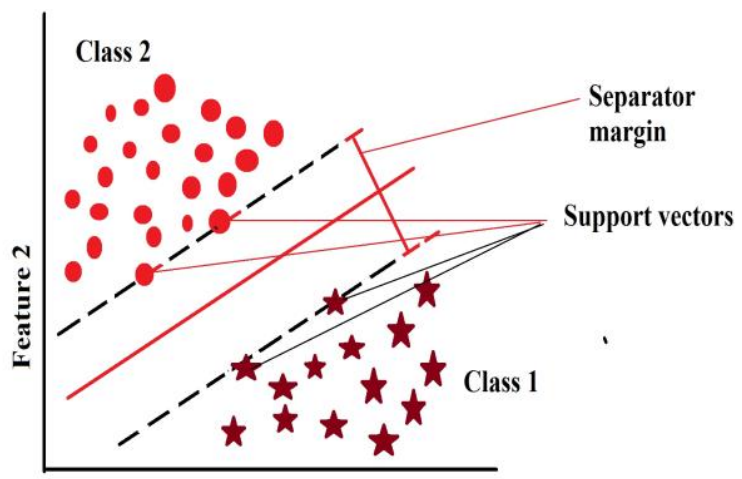

Feature 1

Fig. 5: Support vector machine

In WSN routing is essential to transfer the data from sensor node to sink node. While routing the information eavesdroppers make many attacks to steal the data. In Selective forward attack its decline to forward messages which its gets some times its acts as block hole means when malicious node receive a route request [RREQ] it will send a routing reply [RREP] that indicates route to fraud destinations. S.Kalpanitz, A.Shilton et al (31) in "Detecting selective forward attack in wireless sensor networks using support vector machine" addressed effectively detect black hole and selective forward attack by using band width, hop count, routing information. Outlier also effect the overall performance S.Rajasegarar, C.Leckie et al (32) in "Quarter sphere distributed anomaly detection in WSN" employed method in this one class QSSVM is used, Here Distributed approaches utilized to reduce the communication involving sensor nodes along with computational complexity is squat which reduce the power of sensor nodes.

Intrusion is set of activity that compromises the integrity confidentiality of sensor network. Y.Chen, Y.Qin et al (33) in "intrusion detection based on immune and support vector machine for wireless sensor networks" introduced imperviousness algorithm is a knowledgeable algorithm for complicatedness resolving which was taken from biological immunity systems. Sensor node collected data is first preprocessing through immune algorithm later it will use for SVM for intrusion detection. Furthermore Ellipsoidal one class SVM that can investigate spatial and temporal correlations of the collected readings to detect outlier sensor nodes. SVM techniques are also used to investigate localization and location finding of sensor nodes in wide area. B.Jang, Y.Jang et al (35) in "Area localization algorithm for mobile based wireless sensor networks based on support vector machines" proposed a method by given suitable training data to accomplish goal of localization. This algorithm takes decision metrics like connectivity infor- mation, indicators and fast activities of sensor nodes for localization. By using SVM methods we will improve the efficient learning process for learning algorithms.

\subsection{Bayesian Network}

Bayesian network is probabilistic visual replica that signify situate of arbitrary variables furthermore their conditional addiction. It is mainly used for clustering and classification reason. Planning of Bayesian networks depends on the conditional probability and generates trees based on their probability of occurrence. Compare to other machine learning algorithms Bayesian networks necessitate little number of training samples which makes benefit for wireless sensors which have low power resources but Bayesian utilize present data acquaintance to update earlier to subsequent certainty. By using Bayesian network in wireless sensor we can estimate the event stability using incomplete data sets through investigating past knowledge [9.10.37.38].

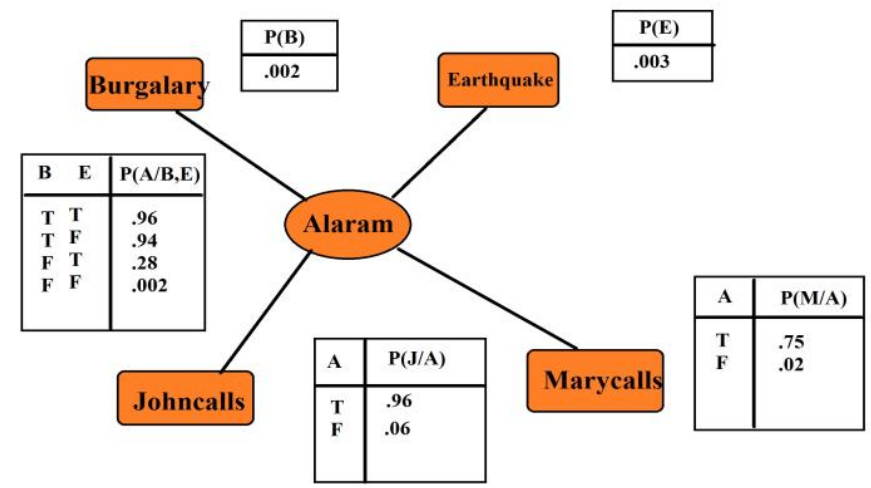

Fig. 6: Bayesian Networks

Bayesian network used in WSN's for activity recognition. P.Zappi, C.Lambriser et al (36) in "Activity Recognition using body sensor" propose a method for detection of body gesture as well as movement. Primarily sensors deployed all over patient body and predict the activity at each sensor node Hidden Markov model applied. Select sensor nodes which collect the most number of gesture information. To get the final gesture decision use Bayesian network model to synthesize the self-regulating node predictions. Bayesian networks also used for localization using only few characteristics of sensor node like radio frequency, received signal angle etc. M.Kim, M.G.Park et al (37) in "Bayesian model network for system energy saving effectiveness for MAC protocols for wireless sensor networks" introduced a scheme uses Bayesian model network for when channel be able to be owed therefore energy is saved.MAC protocol in this method used for supervision active along with sleep times for nodes network rather continually sending observed mechanism we can save network and nodes energy. By using Bayesian belief networks Janakiram, A.Phani kumar et al (38) in "Outlier detection in wsn using Bayesian networks" developed a scheme for outlier detection Bayesian belief networks. Some Sensor node collected information has relative readings which is used to shape conditional probability holding between nodes to determine whether any prospective outliers in the accumulated statistics.

\section{Unsupervised Learning}

Unsupervised learning algorithms no need of training data set. They learn few features from specified data set and apply those features to newly introduced data set. Cataloging data into special status through study the comparisons involving them. Unsupervised algorithms extensively exploit in node clustering as well as data aggregation [9.10.11.12]. 


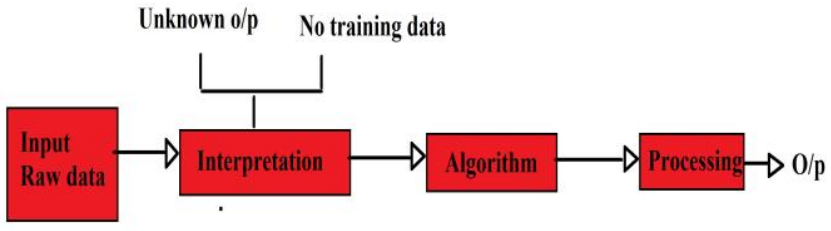

Fig. 7: Unsupervised learning process

\subsection{Principal Component Analysis}

PCA is a dimension diminution and data compression activity that can reduce large amount of data variable to small set data variables that still unmoving the most of the information in large data set. It transforms numeral associated variables into undersized amount of unassociated variables entitled principal components. First component contains highest conflict of the data along with every subsequent component version contains as ample as lasting changeability as achievable. This technique is mostly used in WSN nodes to reduce amount of data transmitting instead of this PCA calculates the uncorrelated components which contains original readings. In event detection, PCA make simpler the problem solving by taking few features from large sensor data set [14.15.16.41].

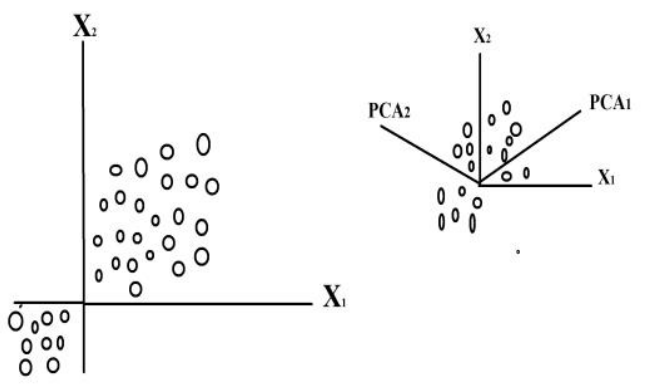

Fig. 8: Principal component analysis

A.Malik et al (41) in "A methodology to optimize query in wireless sensor networks using historical data" presented that a SQL request is sent to the data base and soaring discrepancy components extract from chronological records through PCA. This query data sent to the sensor nodes to extract the sensonary information. Then the original attributes extract from collected information by reversing PCA. Furthermore PCA reduces the transmitted data by finding small set of original readings.PCA methods solve the problem solving by taking small amount of information from large amount of variable in wireless sensor network.

\subsection{K-Means Algorithm}

$\mathrm{K}$ means algorithm used when we have unlabeled data set. $\mathrm{K}$ means algorithm divides data into dissimilar clusters. The items which symbolize equivalent comparable characteristics are put in solitary cluster. It creates $\mathrm{k}$ number of cluster and assigns data points to each $\mathrm{k}$ cluster based on the characteristic that provided. The mean values in particular cluster is the center of that cluster. The k mean algorithm extensively applying in WSN suitable of its easy achievement along with linear complexity. Randomly select $\mathrm{k}$ nodes as centroids for clusters and map each node to nearest centroid with distance measurements. Compute the centroids using current node membership by measuring the threshold value equal to summation of interval amongst nodes plus their respective cen- troids[12.13.42].
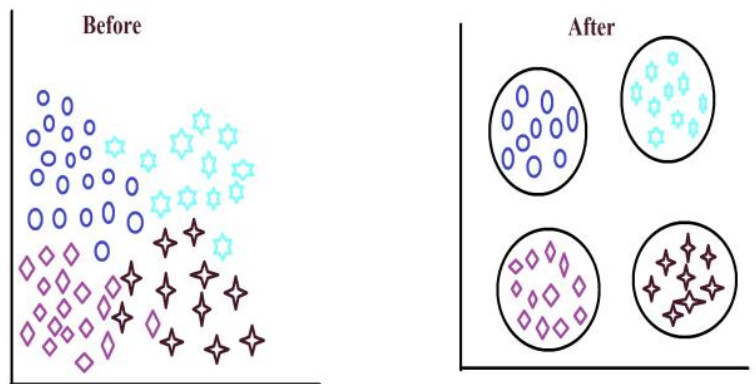

Fig. 9: K Means Algorithm

Tseng et al [42.43] in "integrated surveillance and wireless sensor networks" described about when surveillance collects large amount of data this makes high computation and analysis process. They used iMouse a powerful mobile sensor which divides the sensed data into clusters based on $\mathrm{K}$ means algorithm. $\mathrm{K}$ means algorithm used for object tracking and collecting important information by applying additional learning algorithm. K means algorithm is easiest to implementation and low complexity and it is also sensitive to outliers.

\section{Re-inforcement Learning}

Reinforcement algorithm allows machines, nodes or agent node to discover through cooperating with its surroundings. Machine or agent will find a most excellent decision based on which actions to take so that outcome will more accurate, in order to maximize its performance. This interacting learning process will do for one time or keep going on as environment changes [13.16.45.46.58].

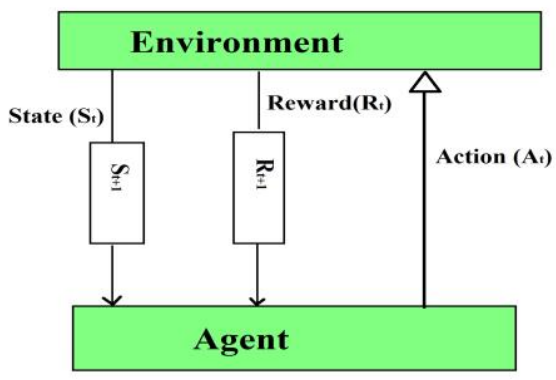

Fig. 10: Reinforcement learning

The most healthy distinguished reinforcement algorithm is Qlearning where agent recurrently updates learning honors supported on the action grabed on particular stipulation.

S.Li, X.Kong et al (44) in "Dynamic path determination of mobile beacons employing reinforcement learning in wireless sensor network" designed a mobile beacon which away from its physical environment while movement. It will determine its position in large number of nodes by using Q-learning process. At particular time each sensor node listen the location update message from mobile beacon node. R.Arroyo, J.Cid et al (45) in "Q probabilistic routing in wireless sensor networks" introduced Geographical routing algorithm where each sensor node finds its routing path by finding which direction-finding path has utmost deliverance tempo in excess of the foregone period time. However above routing protocol remodeled by S.Dong, P.Agarwal, et al (46) in "Reinforcement based geographical routing protocol for UWB wireless networks" applied a protocol and this is different form QPR. In this protocol sensor node finds routing depending upon the value of messages estimated deliverance rate, power limits, message length. Bayesian networks used to find the importance of the message and find the next hop of the message in routing. A .Foster, A.Murphy et al (47) in "Feedback routing for optimizing multiple 
sinks in WSN with reinforcement learning" proposed a narrative procedure which exchange knob regional information as reaction comeback to supplementary sensor nodes. The Q standards are initialized stand on leap or hop tally to apiece node in the set-up. Leap or hop reckoning are calculated by hello messages between sensor node at the time of network deployment. Multicast routing in wireless sensor network each node sends one significance to multiple receivers which consumes power resources of node to avoid this problem. R.Sun, S.Tatsumi et al (48) in "Q-Map multicast routing in wireless sensor networks using reinforcement learning" proposed a Q-Map multicast routing which provides the guarantee reliable resource allocation. In first stage Join query forward discover optimal route and updates the Q values. In Join reply forward stage allow optimal path to multicast transmissions.

\section{Functional challenges}

For designing WSN there are plenty of functional and operational issues are there such as topology changes, power, communication link breakdowns plus memory limitations of node by using machine learning paradigms these types of operational problems are resolved. Machine learning algorithms are productively endorsed to address heterogeneous well-designed challenges in WSN similar to data aggregation and clustering, routing, localization, query processing as well as Medium access control problems.

\subsection{Data aggregation and clustering}

Depending on specific applications sensor nodes may raise to hundreds to thousands for manage the large area. For this outsized sensor network, sending collected data directly to sink node is unsystematic which consume energy of sensor nodes. To overcome these problems surpass data to nearest local aggregator which aggregate all the data from its cluster area and send it to sink node. Many algorithms are presented for optimal election of cluster head which are momentarily described in [58]. The machine learning strategies can develop the advantages of clustering and data aggregation between nodes in a variety of traditions in WSN such as (1) There could be some faulty nodes in WSN's which may possibly produce the faulty or erroneous readings that will distress harmfully on exactness of the whole action of the network. Applying ML we remove faulty sensor nodes. (2) ML methods are engaged to resourcefully decide on the cluster head $(\mathrm{CH})$ and proper $\mathrm{CH}$ selection resolve extensively shrink power expenses along with increase the life span of the network. (3) Machine learning algorithms can effectively compress data locally at cluster heads by efficiently extract important correlations in sensed raw data which will reduce the amount of data to be sent to sink node. Methods used in data aggregation:

* Clustering using neural network in large scale network

* Data aggregation using self-organizing map (SOM)

* Applying learning vector quantization for online data Compression

* Decentralized learning for data latency

* Collaborative data processing through k-means algorithm

* Role-free clustering

* Decide on a cluster head apply decision trees

- Data aggregation using principal component analysis

\subsection{Routing:}

Routing plays vital in send out the data from source node to destination node. In WSN designing a routing protocol consists of a range of prototype obstacles like as data coverage, fault tolerance, energy consumption and scalability. Furthermore sensor nodes endow with limited power resources, compact memory along with knee-high bandwidth. Routing problem is mainly defined as inno- vation the minimum cost path starting at the source vertex and reaching all destination vertices. With help of ML a sensor network is trained from its preceding experience to make optimal direction-finding actions and adapt to the influential atmosphere moreover routing information of sensor nodes exchange with other nodes in the network to find the best optimal routing path. In this process machine learning helps to reduce difficulty of routing algorithm by in view of neighbor nodes information only. Each sensor node autonomously executes routing procedure to decide its path location. Benefits are follows as (1) Proficient to discover optimal routing which turns to prolong life spans of the network and make energy saving of sensor node. (2) Meet the computational complexity models with Quality of Services in routing by using simple classifiers [54].

Some WSN routing protocols which employed in machine learning are specified below:
* Reinforcement learning (RL) for Routing enhancement
* Data routing using self-organizing map
- Distributed regression framework
* Assessment Routing for Optimizing Numerous Sinks in WSN
* Q-Probabilistic Routing

\subsection{Localization:}

Node before sending collected data to nearest neighbor nodes it must know its own location. Nodes are deployed in large geographical area and its makes base station difficult to find node location to provide exact location to the user so it's required of each sensor node to send the location information along with the information collected. Cooperation among nodes is greatly important so that localization can be proficient by themselves not including any human being involvement. In WSN such kind of cooperation can be done between nodes which are located in certain area nodes. Most of the localization algorithms normal, beacon and anchor nodes cooperate with each other to calculate the location information of the node by considering several aspects like limited energy consumption, number and density of nodes and existence of obstacles. Machine learning in localization of WSN recapitulated as follows: In surveillance and object target machine learning divides monitored site into multiple sites to clusters, each cluster represents specific location indicator. Using fixed anchor points we can eliminate range of measurements [49.50.53].

Some suitable stratagems for WSN localization using Machine learning

Decision tree based localization
Path determination using reinforcement learning
Lensor placements through Gaussian processes
Localization using self-organizing map
Localization based on neural network
Spatial Gaussian process regression
Lobulization using support vector regression
Localization using support vector machine
Bayesian node localization

\subsection{Query processing:}

Query processing and Event detection are measured to be welldesigned necessities of any huge extent sensor network. In recently many researchers takes attention on design of effective query processing and event detection. The simplest technique for query processing and event detection is making a crucial strict threshold value but in most of WSN applications query and event entities are difficult plus entail more than one threshold value. Machine 
learning techniques expand highly developed query processing and event detection solution strategies in WSN. Some WSN Query processing's which employed in machine learning are specified below:

* Forest fire detection with neural network

* Query optimization using principal component analysis

* Event recognition through Bayesian algorithms

* Query processing through k-nearest neighbors

* Disseminated event discovery for disaster management using decision tree

\subsection{Medium Access Control:}

In WSNs, huge amount of sensors collaborate to proficiently transmit data. Hence, constructing MAC protocols for WSNs creates dissimilar disputes from distinctive wireless networks as well as energy exhaustion and discontinuation. ML mostly exploit to attentively decide duty cycle of a node via broadcast history of the network means sleep in mean time as well as wake up when channel is estimated to be inactive. Attain secured data communication by unite the perception of machine learning along with MAC protocols [56.57]. Some suitable stratagems for WSN MAC protocol using Machine learning:

Neural network-based MAC
Adaptive MAC layer
Bayesian statistical model for MAC
Duty cycle management using reinforcement learning
Adaptive MAC layer

\section{Non-functional challenges}

Operational challenges directly related with basic operational or functional behavior of systems with WSN while non-operational aspects not associated with basic operation of the system. In WSN non functional challenges are Quality of services, security and data integrity.

\subsection{Quality of Services and data Integrity:}

QoS undertaking huge priority to delivery of real-time actions as well as data. In the contexts of WSN's there is probable multi-hop transmission of data to end user. WSN's agonize with bandwidth and power constraints that can restrict the amount of information which can be convoyed from a source to destination node. Moreover data dissemination and data aggregation in WSN's can be faulty and unreliable. Data integrity, QoS guarantee plus error detection depends on the application and network services. ML mechanisms are proficient to handle much of these while guaranteeing valuable resources mainly power consumption plus bandwidth [52.55]

\section{* QoS provisioning using reinforcement learning \\ * QoS estimation using neural network \\ * Assessing truthfulness and steadfastness of sensor nodes using multi-output Gaussian processes \\ * Metric Map (link quality estimation framework)}

\subsection{Security and Intrusion Detection:}

Wireless sensor networks deployed in a rough environment where human can't properly work. In that environment traditional networks are also failed to deployment. Every sensor network collects the information and sends it to desirable sink node or base station. All the data not available to everyone some information is confidential which only accessible through a secure channel. The key dispute to implement security procedures in WSN's is the restricted resources constraints. Additionally a few encounter methods intent to construct unanticipated, erroneous data by introducing disingenuous interpretation to the network [51].

Machine learning algorithms have been engaged to discover outlying and disingenuous dimensions. Machine learning in WSN's provides some earnings those follows as (1) Online learning and prevention of malicious attacks and vulnerabilities. (2) Preventing misleading and transmission of outlier save nodes energy and significantly increase the lifespan of the network. (3) Eliminating faulty misleading readings enhance the network reliability. Some suitable stratagems for WSN Security using Machine learning

* Deviation revealing using k-nearest neighbors

* Evaluating intrusions with self-organizing map

* Identify selective forwarding attacks with support vector machine

* Anomaly uncovering through k-nearest neighbors

* Bayesian belief network approach for Outlier detection

\section{Conclusion}

WSN completely peculiar from time-honored networks in a variety of components moreover WSN's require various innovative solutions for anomaly recognition, reliability, scheduling, QoS, clustering, routing techniques, data collection, localization. Machine learning maintains a compilation of methods to progress the ability of WSN's. Many design challenges in WSN resolve with various ML techniques. Inside this article we provided literature survey of recent works done in WSN moreover we discussed various kinds of machine learning approaches particularly used in WSN challenges along with operational and non operational issues in WSN. More work has to be done to expand lightweight distributed message transient procedures, energy resource management problem of WSN.

\section{References}

[1] I.F.Akykidze, W.Su, Y.Sankarasubramaniam, "Wireless sensor networks A Survey", Computer Networks, Volume 38,Issue 4, March 2002 , IEEE.

[2] K. Romer, F. Mattern, "The design space of wireless sensor networks", IEEE Wireless Commun., vol. 11, no. 6, pp. 54-61, Dec. 2004

[3] D. E. D. Culler, and M. Srivastava, "Overview of sensor networks", IEEE Computer, pp. 41-49, 2004

[4] Shabbir Hasan, R.K. Singh, "A Survey of Wireless sensor Networks", International journal of Emerging Technology and Advanced Engineering, Volume 3, Issue 3, March 2013

[5] Sanjeev Kumar Guptha, Poonam Sinha, "Overview of wireless sensor networks: A Survey", International Journal of Advanced Research in Computer and Communication Engineering, Volume 3, Issue 1, January 2014

[6] Neha Singh, Kamakshi Rautela, "Literature Survey on Wireless sensor networks", International journal of Engineering and computer Science, Volume 5, Issue 8, August 2016

[7] Mitchell, "Machine Learning", McGraw-Hill, 1997

[8] M. Welling, "A First Encounter with Machine Learning"

[9] T. O. Ayodele, "Introduction to machine learning" in New Advances in Machine Learning, Rijeka, Croatia:InTech, 2010

[10] P. Harrington, "Machine Learning in action", Manning Publications Co. Shelter Island, New York, 2012

[11] Ayon Dey, "Machine Learning Algorithms: A Review", International Journal of Computer Science and Information Technologies (IJCSIT), Vol. 7 (3) , 2016, 1174-1179

[12] M. Di, E. M. Joo, "A survey of machine learning in wireless sensor networks from networking and application perspectives", Proc. 6th Int. Conf. Inf. Commun. Signal Process., pp. 1-5, 2007

[13] Jennifer Yick, Biswanath Mukherjee, Dipak Ghosal, "Wireless sensor network survey," Computer Networks Elsevier 52 , pp 2292-2330, 2008

[14] Romer, Mattern, "The Design Space of Wireless Sensor Networks", IEEE Wireless Communication, 2004

[15] Forster, "Machine learning techniques applied to wireless adhoc networks: Guide and survey", In Proceedings of the 3rd International Con- 
ference on Intelligent Sensors, Sensor Networks and Information Processing (ISSNIP), ISSNIP

[16] S. R. K. Joel B. Predd, and H. Vincent Poor, "Distributed Learning in Wireless Sensor Networks - application issues and the problem of distributed inference", IEEE Signal Processing Magazine, 2006

[17] A. Mainwaring, D. Culler, J. Polastre, R. Szewczyk, and J. Anderson, "Wireless sensor networks for habitat monitoring", in Proceedings of the 1st ACM International Workshop on Wireless Sensor Networks and Applications, ACM, 2002, pp. 88-97

[18] E.S. Nadimia,b, H.T. Søgaardc, T. Bak, "ZigBee-based wireless sensor networks for classifying the behavior of a herd of animals using classification trees", biosystem engineering, Science direct,100 (2008) 167176

[19] Z.Meri, M.Bayoumi et al, "A lightweight fault tolerant target location system for wireless sensor networks", international journal for computing, 2012,pp 234-238

[20] E.Cayiric, Y.Dogan et al, "wireless sensor networks for underwater surveillance", Elsevier B.V , Ad Hoc Networks 4 (2006) 431-446.

[21] J. W. Branch, C. Giannella, B. Szymanski, R. Wolff, and H. Kargupta, "In-network outlier detection in wireless sensor networks" Knowledge and information systems, vol. 34, no. 1, pp. 23-54, 2013

[22] J. Winter, Y. Xu, and W.-C. Lee, "Energy efficient processing of k nearest neighbor queries in location-aware sensor networks", in 2nd International Conference on Mobile and Ubiquitous Systems: Networking and Services, IEEE, 2005, pp. 281-292

[23] P. P. Jayaraman, A. Zaslavsky, and J. Delsing, "Intelligent processing of k-nearest neighbors queries using mobile data collectors in a location aware 3D wireless sensor network," in Trends in Applied Intelligent Systems. Springer, 2010, pp. 260-270

[24] Dan Li, Kerry D. Wong, Detection, "Classification and Tracking of Targets in Distributed Sensor Networks", IEEE Signal processing, march, 2002

[25] L. Yu, N. Wang, and X. Meng, "Real-time forest fire detection with wireless sensor networks", in International Conference on Wireless Communications, Networking and Mobile Computing, vol. 2, 2005, pp. 1214-1217

[26] O. Postolache, J. Pereira, and P. Girao, "Smart sensors network for air quality monitoring applications", IEEE Transactions on Instrumentation and Measurement, vol. 58, no. 9, pp. 3253-3262, 2009

[27] Y. Gao, Y. Lin, and Y. Sun, "A wireless sensor network based on the novel concept of an I-matrix to achieve high-precision lighting control", Building and Environment, vol. 70, pp. 223-231, 2013

[28] A. Snow, P. Rastogi, and G. Weckman, "Assessing dependability of wireless networks using neural networks", in Military Communications Conference, IEEE, 2005, pp. 2809-2815 Vol. 5

[29] Raghavendra V. Kulkarni ; Ganesh K. Venayagamoorthy, "Neural network based secure media access control protocol for wireless sensor networks", International Joint Conference on Neural Networks, 14-19 June 2009

[30] Y.J. Shen and M.-S. Wang, "Broadcast scheduling in wireless sensor networks using fuzzy Hopfield neural network", Expert Systems with Applications, vol. 34, no. 2, pp. $900-907,2008$

[31] S. Kaplantzis, A. Shilton, N. Mani, and Y. Sekercioglu, "Detecting selective forwarding attacks in wireless sensor networks using support vector machines", in 3rd International Conference on Intelligent Sensors, Sensor Networks and Information. IEEE, 2007, pp. 335-340

[32] S. Rajasegarar, C. Leckie, M. Palaniswami and J. Bezdek, "Quarter sphere based distributed anomaly detection in wireless sensor networks", in International Conference on Communications, 2007, pp. 3864-3869

[33] Y. Chen, Y. Qin, Y. Xiang, J. Zhong, and X. Jiao, "Intrusion detection system based on immune algorithm and support vector machine in wireless sensor network", in Information and Automation, ser. Communications in Computer and Information Science. Springer, Berlin Heidelberg, 2011, vol. 86, pp. 372-376

[34] S. Kaplantzis, A. Shilton, N. Mani, and Y. Sekercioglu, "Detecting selective forwarding attacks in wireless sensor networks using suppor vector machines", in 3rd International Conference on Intelligent Sensors, Sensor Networks and Information. IEEE, 2007, pp. 335-340

[35] Bin Yang, Jianhong Yang, Jinwu Xu, Debin Yang, “Area Localization Algorithm for Mobile Nodes in Wireless Sensor Networks Based on Support Vector Machines", International Conference on Mobile Ad-Hoc and Sensor Networks Mobile Ad-Hoc and Sensor Networks pp 561-571

[36] P. Zappi, C. Lombriser, T. Stiefmeier, E. Farella, D. Roggen, L. Benini, and G. Tröster, "Activity recognition from on-body sensors: Accuracy power trade-off by dynamic sensor selection", in Wireless Sensor Net works. Springer, 2008, pp. 17-33

[37] J.-M. Kim, S.-H. Park, Y.-J. Han, and T.-M. Chung, "CHEF: Cluster head election mechanism using fuzzy logic in wireless sensor networks", in 10th International Conference on Advanced Communication Technology, vol. 1. IEEE, 2008, pp. 654-659

[38] D. Janakiram, V. Adi Mallikarjuna Reddy, and A. Phani Kumar, "Outlier detection in wireless sensor networks using Bayesian belief networks", in 1st International Conference on Communication System Software and Middleware. IEEE, 2006, pp. 1-6
[39] M. Morelande, B. Moran, and M. Brazil, "Bayesian node localization in wireless sensor networks", in IEEE International Conference on Acoustics, Speech and Signal Processing, 2008, pp. 2545-2548

[40] D. Feldman, M. Schmidt, C. Sohler, D. Feldman, M. Schmidt, and C. Sohler, "Turning big data into tiny data: Constant-size coresets for kmeans, PCA and projective clustering", in SODA, 2013, pp. 1434-1453

[41] H. Malik, A. Malik, and C. Roy, "A methodology to optimize query in wireless sensor networks using historical data", Journal of Ambient Intelligence and Humanized Computing, vol. 2, pp. 227-238, 2011

[42] I. T. Jolliffe, "Principal component analysis". Springer verlag, 2002

[43] Y.-C. Tseng, Y.-C. Wang, K.-Y. Cheng and Y.-Y. Hsieh, "iMouse: An integrated mobile surveillance and wireless sensor system", Computer applications, vol. 40, no. 6, pp. 60-66, 2007

[44] S. Li, X. Kong, and D. Lowe, "Dynamic path determination of mobile beacons employing reinforcement learning for wireless sensor localization", in 26th International Conference on Advanced Information Net working and Applications Workshops, 2012, pp. 760-765.

[45] R. Arroyo-Valles, R. Alaiz-Rodriguez, A. Guerrero-Curieses, and J. Cid-Sueiro, "Q-probabilistic routing in wireless sensor networks", in 3rd International Conference on Intelligent Sensors, Sensor Networks and Information. IEEE, 2007, pp. 1-6

[46] S. Dong, P. Agrawal, and K. Sivalingam, "Reinforcement learning based geographic routing protocol for UWB wireless sensor network," in Global Telecommunications Conference. IEEE, 2007, pp. 652-656

[47] A. Förster and A. Murphy, "FROMS: Feedback routing for optimizing multiple sinks in wsn with reinforcement learning", in 3rd International Conference on Intelligent Sensors, Sensor Networks and Information. IEEE, 2007, pp. 371-376.

[48] R. Sun, S. Tatsumi, and G. Zhao, "Q-MAP: A novel multicast routing method in wireless ad hoc networks with multiagent reinforcement learning", in Region 10 Conference on Computers, Communications, Control and Power Engineering, vol. 1, 2002, pp. 667-670 vol.1

[49] A. Shareef, Y. Zhu, and M. Musavi, "Localization using neural networks in wireless sensor networks," in Proceedings of the 1st International Conference on Mobile Wireless Middleware, Operating Systems, and Applications, 2008, pp. 1-7

[50] A. Snow, P. Rastogi, and G. Weckman, "Assessing dependability of wireless networks using neural networks", in Military Communication Conference. IEEE, 2005, pp. 2809-2815 Vol. 5

[51] A. Moustapha and R. Selmic, "Wireless sensor network modeling using modified recurrent neural networks: Application to fault detection", IEEE Transactions on Instrumentation and Measurement, vol. 57, no. 5, pp. 981-988, 2008

[52] Y. Wang, M. Martonosi, and L.-S. Peh, "Predicting link quality using supervised learning in wireless sensor networks", ACM SIGMOBILE Mobile Computing and Communications Review, vol. 11, no. 3, pp. 7183,2007

[53] G. Giorgetti, S. K. S. Gupta, and G. Manes, "Wireless localization using self-organizing maps", in Proceedings of the 6th International Conference on Information Processing in Sensor Networks, ser. IPSN'07. New York, NY, USA: ACM, 2007, pp. 293-302

[54] S. Li, X. Kong, and D. Lowe, "Dynamic path determination of mobile beacons employing reinforcement learning for wireless sensor localization", in 26th International Conference on Advanced Information Networking and Applications Workshops, 2012, pp. 760-765

[55] X. Liang, M. Chen, Y. Xiao, I. Balasingham, and V. C. M. Leung, "A novel cooperative communication protocol for QoS provisioning in wireless sensor networks", in 5th International Conference on Test bed and Research Infrastructures for the Development of Networks Communities and Workshops, 2009, pp. 1-6

[56] Y. Chu, P. Mitchell, and D. Grace, "ALOHA and q-learning based medium access control for wireless sensor networks", in International Symposium on Wireless Communication Systems, 2012, pp. 511-515

[57] K. Klues, G. Hackmann, O. Chipara, and C. Lu, "A component based architecture for power-efficient media access control in wireless sensor networks", in Proceedings of the 5th International Conference on Embedded Networked Sensor Systems. ACM, 2007, pp. 59-72

[58] M. Seah, C.-K. Tham, V. Srinivasan, and A. Xin, "Achieving coverage through distributed reinforcement learning in wireless sensor networks", in 3rd International Conference on Intelligent Sensors, Sensor Networks and Information. IEEE, 2007, pp. 425-430 